Comparison between two photovoltaic module models based on transistors

Frédéric Saint-Eve, Jean-Paul Sawicki, Pierre Petit, Fabrice Maufay, and Michel Aillerie

Citation: AIP Conference Proceedings 1968, 030082 (2018); doi: 10.1063/1.5039269

View online: https://doi.org/10.1063/1.5039269

View Table of Contents: http://aip.scitation.org/toc/apc/1968/1

Published by the American Institute of Physics 


\title{
Comparison between Two Photovoltaic Module Models Based on Transistors
}

\author{
Frédéric Saint-Eve ${ }^{1,2, a)}$, Jean-Paul Sawicki ${ }^{1,2, b)}$, Pierre Petit ${ }^{1,2}$, \\ Fabrice Maufay ${ }^{1,2}$, and Michel Aillerie ${ }^{1,2}$ \\ ${ }^{1}$ Université de Lorraine, LMOPS-EA 4423, IUT de Thionville-Yutz, 57970 Yutz, France. \\ ${ }^{2}$ CentraleSupelec, LMOPS-EA 4423, 57070 Metz, France. \\ a)Corresponding author: frederic.saint-eve1@ac-nancy-metz.fr \\ b) jean-paul.sawicki@univ-lorraine.fr
}

\begin{abstract}
The main objective of this paper is to verify the possibility to reduce to a simple electronic circuit with very few components the behavior simulation of an un-shaded photovoltaic (PV) module. Particularly, two models based on well-tried elementary structures, i.e., the Darlington structure in first model and the voltage regulation with programmable Zener diode in the second are analyzed. Specifications extracted from the behavior of a real I-V characteristic of a panel are considered and the principal electrical variables are deduced. The two models are expected to match with open circuit voltage, maximum power point (MPP) and short circuit current, without forgetting realistic current slopes on the both sides of MPP. The robustness is mentioned when irradiance varies and is considered as an additional fundamental property. For both models, two simulations are done to identify influence of some parameters. In the first model, a parameter allowing to adjust current slope on left side of MPP proves to be also important for the calculation of open circuit voltage. Besides this model does not authorize an entirely adjustment of I-V characteristic and MPP moves significantly away from real value when irradiance increases. On the contrary, the second model seems to have only qualities: open circuit voltage is easy to calculate, current slopes are realistic and there is perhaps a good robustness when irradiance variations are simulated by adjusting short circuit current of PV module. We have shown that these two simplified models are expected to make reliable and easier simulations of complex PV architecture integrating many different devices like PV modules or other renewable energy sources and storage capacities coupled in parallel association.
\end{abstract}

\section{INTRODUCTION}

These last years, the need of electrical energy is continuously increasing, especially the need of "green" electricity with no - or - less pollution, and the need of production in some distant places where there may not be electrical network possibilities. In this context, photovoltaic modules are a very good solution, and have been studied for a long time, with different models used for simulations: single diode [1-3], double diode [4], and MOSFET- based [5], with for some models, the consideration of real climate conditions adding numerous parameters. A major point of these simulations is the straightforward relation existing between the complexity of the model and the necessary computation time [1], especially when the simulation includes the converter and the charge circuitry. Particularly, and as example, we consider the parallel architecture allowing integration of PV modules connected to individual converters designed to step up voltage enough to supply grid inverters [6]. With the aim to provide a complete solution to realize DC micro-grids dedicated to smart grid approach, individual storage capacities have to work connecting to the same DC power bus, when various laws and different algorithms are implemented in converters [7]. To predict behavior of parallel architecture in the case of transient phenomena like drop of irradiance, partial or complete shading on PV modules or rough disturbances due to loads on power bus, simulation software seems to be an efficient tool to generate reliable results, especially when the software 
environment (Proteus in our case) used for the development is also able to simulate programs implemented in the microcontroller of converter (as for the tracking of the Maximum Power Point (MPPT), various regulations, diagnosis routines, or communication with a supervisor and so on). We can easily imagine the workload for simulation software in this case. For these reasons, original PV module models are developed in order to present faster in simulation with good reliability compared to the experimental measurements. Objective of this paper is to compare two of them, based on well-tried elementary structures, i.e., the Darlington structure in first model and the voltage regulation with programmable Zener diode in the second, and connected to elementary load before applying them to step up converters [8].

\section{EXPERIMENTAL I-V CHARACTERISTIC OF A PV PANEL}

\section{Experimental Bench}

\section{Photovoltaic (PV) Modules}

An indoor low power PV module is preferred to a roof module for convenience. An artificial illuminator composed of six halogen lamps (electrical power equal to $6 \mathrm{~kW}$ ) is used to easily adjust irradiance being measured thanks to a pyrometer. Table 1 displays specifications of the low voltage module, in polycrystalline silicon technology, experimentally used in this study for validation of the modeling results.

TABLE 1. PV module specifications $\left(\mathrm{AM}=1.5, \theta=25^{\circ} \mathrm{C}\right)$

\begin{tabular}{lc}
\hline \multicolumn{1}{c}{ Electrical Data } & Polycrystalline silicon technology \\
\hline Peak Power (W) & 50 \\
Open Circuit Voltage (V) & 21.8 \\
Short Circuit Current (A) & 3.2 \\
MPP Voltage (V) & 17.5 \\
MPP Current (A) & 2.9 \\
\hline
\end{tabular}

\section{MCB Converter}

A Magnetically Coupled Boost (MCB) converter is used to extract the maximum power of the PV module at a given output voltage. It is mainly composed by a MOSFET transistor with low $\mathrm{R}_{\mathrm{DSon}}$ [9] and by an autotransformer, designed to reach significant DC voltages, higher than $100 \mathrm{~V}_{\mathrm{DC}}$. It is to note that this converter is not insulated because of a recovery diode connected between the primary and secondary windings. A natural, but low current appears at the output of converter when PV module is illuminated, even if the MOSFET transistor is not controlled. So, the measured voltage is not truly Open Circuit Voltage. A simple slope is applied on duty cycle control, between 0 and $97 \%$. To ensure the safety of the converter, input voltage and current, like output voltage measured by internal sensors are used in control program. Indeed, in the sizing of the converter and of its control program, we have considered that the output voltage will be limited to $250 \mathrm{~V}$, assumed by a voltage divider bridge and that the input current must remain below $6 \mathrm{~A}$, limited by the linearity of the shunt resistors. This sizing was designed for a full PV plan as we can find on a house roof even if the chosen PV module is unable to deliver such current. All internal measures could be kept for I-V characteristic generation but another specific device is preferred to acquire electrical variables. Finally, pulse width modulation (PWM) control is realized by a Microchip PIC 18F252 microcontroller.

\section{Loads Connected to MCB Converter}

The role of loads connected to the output of the MCB converter is to dissipate all the power delivered by the PV module. Thus, for a given irradiation and temperature and thanks to the PWM control, this power can be adjusted and will reach the maximum power point (MPP) of the PV module for a specific value of the duty cycle. At MPP, the output voltage is maximal and naturally loads have to be suitable to this voltage. Incandescent lamps are preferred in our experiment to pure resistors, energy-saving lamps or leds because they can dissipate significant power in a small volume and because they are designed to work under standard high voltage $(230 \mathrm{~V})$. If halogen illuminator could be able to realize Standard Test Conditions (1000 W/m2 with solar spectrum), incandescent lamps 
would be chosen to dissipate around $50 \mathrm{~W}$. So, two lamps, one of $25 \mathrm{~W}$, the second of $40 \mathrm{~W}$, were connected in parallel to the converter output provided a maximum of $65 \mathrm{~W}$ dissipated power. Besides, use of low power lamp allows the power bus to reach significant voltage even if irradiance is not at its maximum.

\section{Signal acquiring system}

As seen above, the acquisition of internal PV current and voltage could be dedicated to the microcontroller driving the converter. But with consideration of the available memory of the Microchip PIC 18F252 microcontroller, only few points, around one hundred, could be saved inducing a slope of duty cycle with a step around $1 \%$ per point. It results that with a temporization of 10 milliseconds between each point, I-V characteristic could be described in only 1 second. Nevertheless, to minimize transient effects between PV module and converter it was decided to modify the slope of the duty cycle, thus reducing the step to only $0.1 \%$ per point. Within this solution and by keeping the same delay between points, the scanning of the I-V characteristic will need 10 seconds. This duration is considered low enough to remain the converter behavior undisturbed by an increase of the PV module temperature.

Based on the previous considerations, the requirement of memory storage is estimated to two thousand measures for the PV module current and voltage, which exceeds the microcontroller capacity, so a more powerful system is required. In order to solve this problem, we have developed a topology based on a commercial USB acquiring module (LabJack U3-LV) and dedicated cards for the conditioning of the analog signals. With Hall effect sensors for the currents of module and bus and opto-couplers for voltage, this topology of system allows interesting technical performances, especially when are considered the associated analog to digital resolution of 12 bits and the temporal resolution with a sample period up to $1 \mathrm{~ms}$. Furthermore, thanks to the important storage capacity of computer connected via USB to the controller, others variables like output current or voltage can be easily acquired.

A virtual measurement application, with possibility to save measurements for further treatment was easily developed thanks to Profilab Expert software. Finally, in terms of performance, the sequential scanning of the various I-V characteristics with a period equal to 1 millisecond needs, during our experiments, ten thousand measurements per electrical variable.

\section{Experimental Curves}

\section{I-V and P-V characteristics}

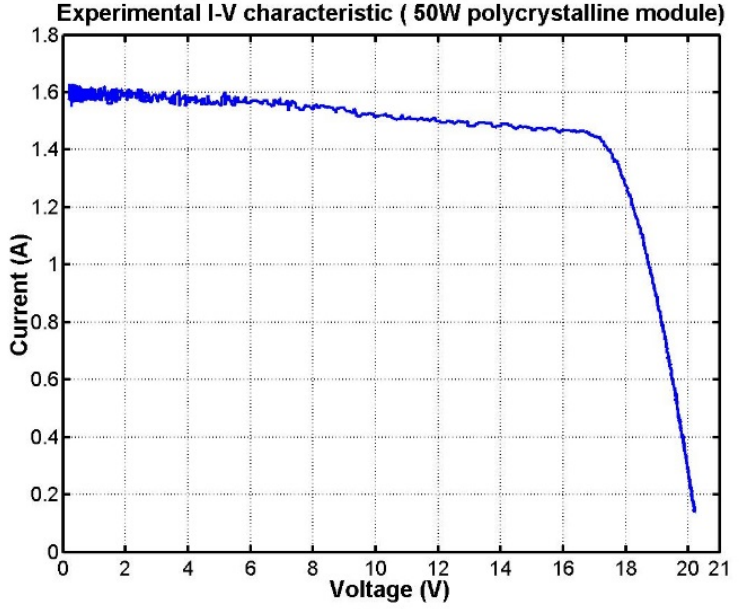

(a)

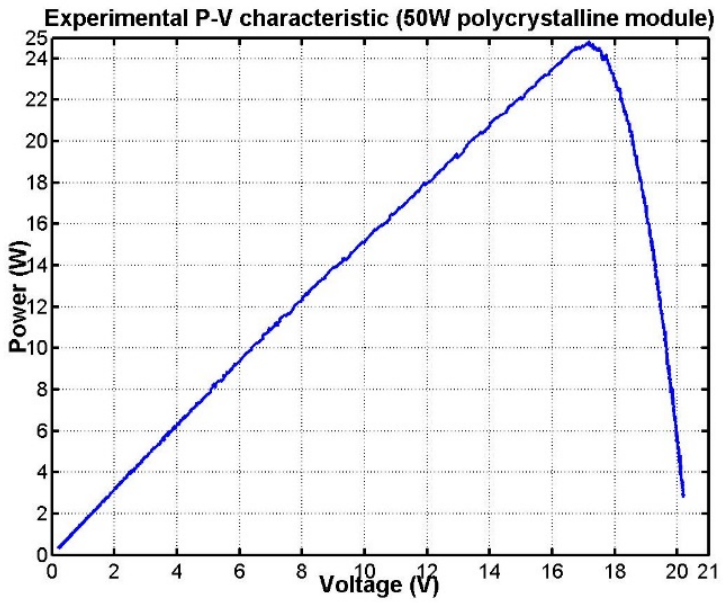

(b)

FIGURE 1. Scanning of I-V characteristic during 10 seconds (a). Computing of P-V characteristic (b).

All data saved on the Personal Computer are treated by the well-known software Matlab. Figure 1.a shows that the open circuit voltage cannot be measured because of natural conduction between primary and secondary windings when the MOSFET is in its open mode. Nevertheless it can be extrapolate graphically to around $20.4 \mathrm{~V}$. It is to notice that this curve is obtained under an irradiation measured by a pyrometer equal to $1403.7 \mathrm{~W} / \mathrm{m}^{2}$ with a surface 
temperature around $20{ }^{\circ} \mathrm{C}$ for PV module. But we found that the short circuit current is only half of the current expected for Standard Test Conditions $\left(1000 \mathrm{~W} / \mathrm{m}^{2}, 25^{\circ} \mathrm{C}\right)$. We can conclude that the spectrum of the halogen lamps differs significantly from solar spectrum. Figure 1 (b) confirms that PV module supplies only half of its theoretical peak power, precisely $24.83 \mathrm{~W}$. So it is possible to deduce voltage $\left(\mathrm{U}_{\mathrm{MPP}}=17.20 \mathrm{~V}\right)$ and current $\left(\mathrm{I}_{\mathrm{MPP}}=1.44 \mathrm{~A}\right)$ at this point. These real electrical values will become the reference specifications for the two models below.

\section{MODEL BASED ON TWO DARLINGTON CIRCUIT-MOUNTED TRANSISTORS}

\section{Electrical Diagram}

\section{Description}

Modeling PV modules in real conditions has to consider generally at minima three strings of PV cells, taking account of role of bypass diodes dedicated to hot spot avoiding. So, previous studies have shown how associate structures based on bipolar transistor to simulate behavior of complete PV module, with homogeneous irradiance like partially shading [10]. In this paper we model a low power PV module without any bypass diode. So we prefer to simulate a model based on Darlington structure, which has been purposed in recent paper [11]. As shown in Fig. 2 photo-generator current is realized by a current source equal to short circuit current $\left(\mathrm{I}_{1}\right)$. When load $\left(\mathrm{I}_{2}\right)$ does not dissipate any current (open circuit state), all the sources of current $I_{1}$ has to be bypassed. The greater part is due to conduction of power bipolar transistor (2N3055), the rest in the parallel resistor (Rp), in the collector of control bipolar transistor (BCW60C) and in the voltage divider bridge $\mathrm{R}_{1}-\mathrm{R}_{2}$.

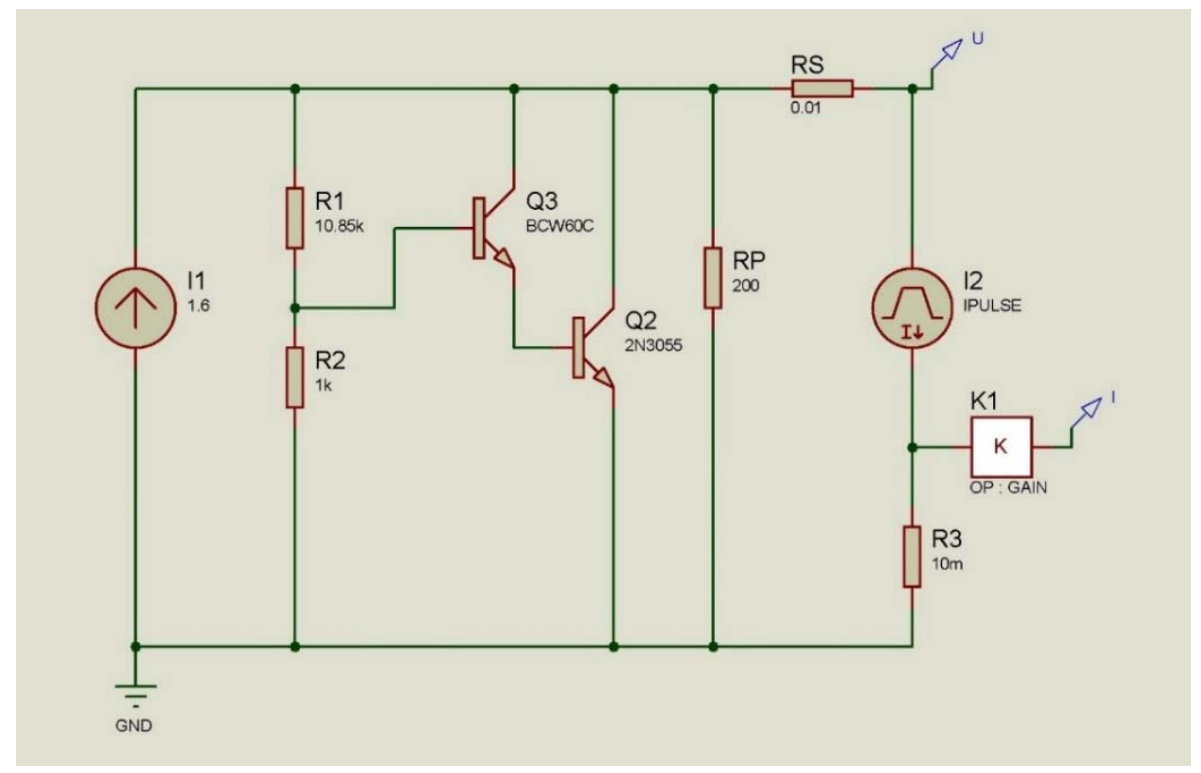

FIGURE 2. Model based on Darlington structure.

\section{Open circuit voltage calculation}

In the following we suppose the parallel resistance Rp very high and neglecting for a while voltage divider bridge $R_{1}-R_{2}$, thus, as a first approximation, all the short circuit current $I_{1}$ is bypassed by the power transistor (2N3055). Specifications in datasheet give a current gain equal to 20 , so base current is equal to $80 \mathrm{~mA}$ being quasi the same that the collector current of the low power transistor (BCW60C). We can verify in datasheet that this value is in conformity with maximal value $(100 \mathrm{~mA})$. A current gain is done for this transistor, 90 , when collector current is equal to $50 \mathrm{~mA}$. Even if real current is higher than datasheet value we decide to keep this current gain. We obtain 
a base current equal to $0.9 \mathrm{~mA}$. It is to notice that the choice of BCW60C in the setup would not allow to exceed $2 \mathrm{~A}$ for short circuit current, keeping always a current gain equal 20.

As voltage of resistor $\mathrm{R}_{2}$ is equal to $2 *$ Vbe (between 1.3 and $1.4 \mathrm{~V}$ ) it is easy to deduce the current crossing through $R_{2}$, especially when $R_{2}$ equal to $1 \mathrm{k} \Omega$. As current in $R_{1}$, named $I_{R 1}$ in equation (1) is the sum of the base current and the $R_{2}$ current, the result is $2.2 \mathrm{~mA}$. So resistance $\mathrm{R}_{1}$ can be deduced when open circuit voltage Uoc is set, by:

$$
R 1=\frac{U o c-2 * V b e}{I R 1}
$$

As previously mentioned, we have neglected the parallel resistance Rp. In fact, infinite resistance does not allow to obtain realistic I-V curve because there is no increasing of current on the left side of MPP. So, introduction of lower parallel resistance will decrease the open circuit voltage because the power transistor bypasses less current with consequence of less current in the voltage divider bridge. In this case, it will be necessary to increase $\mathrm{R}_{1}$ when decreasing Rp to keep the same Uoc, which needs few successive simulations.

\section{Simulations with Different Parameters}

\section{Setting of $R_{p}$ resistance}

Two simulated I-V characteristics are shown with Rp equal to $200 \Omega$ in Fig. 3.a and with Rp equal to $100 \Omega$ in Fig. 3.b; the serial resistance Rs has been chosen at a very low value equal to $0.01 \Omega$.

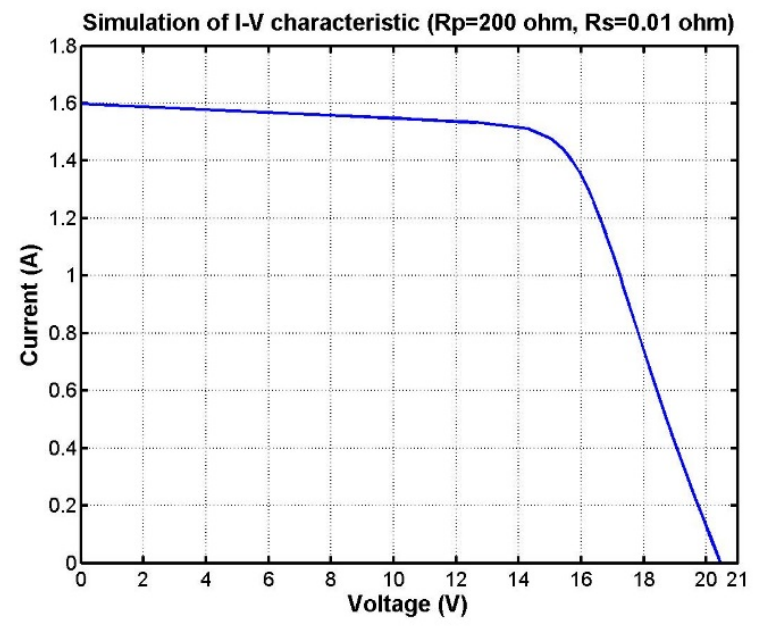

(a)

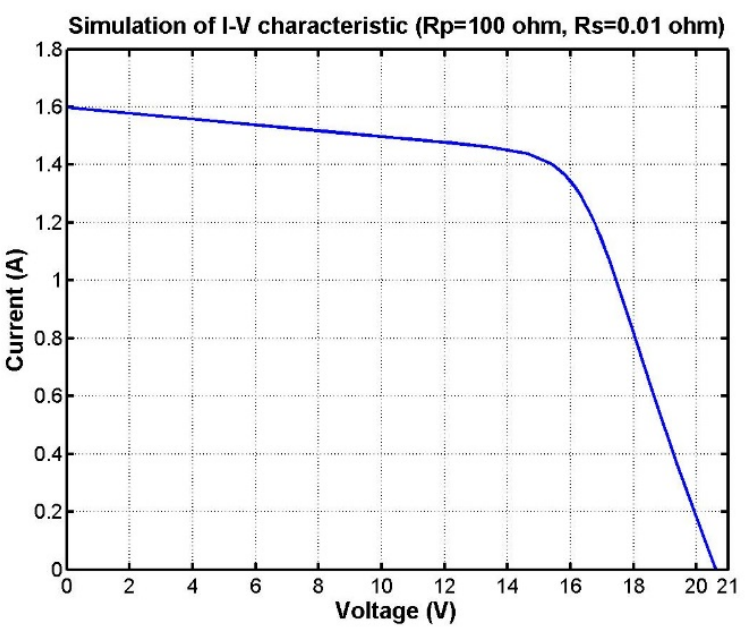

(b)

FIGURE 3. Simulation with parallel resistance equal to $200 \Omega$ (a) and with parallel resistance equal to $100 \Omega$ (b).

Virtual scanning of PV module is realized by a load ( $\mathrm{I}_{\text {pulse }}$ in Fig. 2$)$ which dissipates the current varying linearly from zero to its maximum value equal to the short circuit current, i.e. $1.6 \mathrm{~A}$ during 0.1 second. From the open circuit point to the MPP, the transistor bypasses a current which decreases, explaining the fall of the module voltage due to the decrease of the base current, then due to the bridge divider current. In this simulation, influence of serial resistance Rs is negligible. From the MPP to the short circuit point, the power transistor is not in conduction mode and the PV module voltage straightforwardly depends on the current passing through the parallel resistance. If this resistance is infinite, there is no current and the short circuit current is reached very quickly, implying horizontal curve. In other cases, when Rp exists, the decreasing of the PV module voltage is simply explained by Ohm law as the resistor initially dissipates a relatively important current which naturally decreases as current load approaches the short circuit point. Thus, the slope of the current on the left side of the MPP depends on the initial current in Rp equal to the difference between short circuit value and MPP current. 


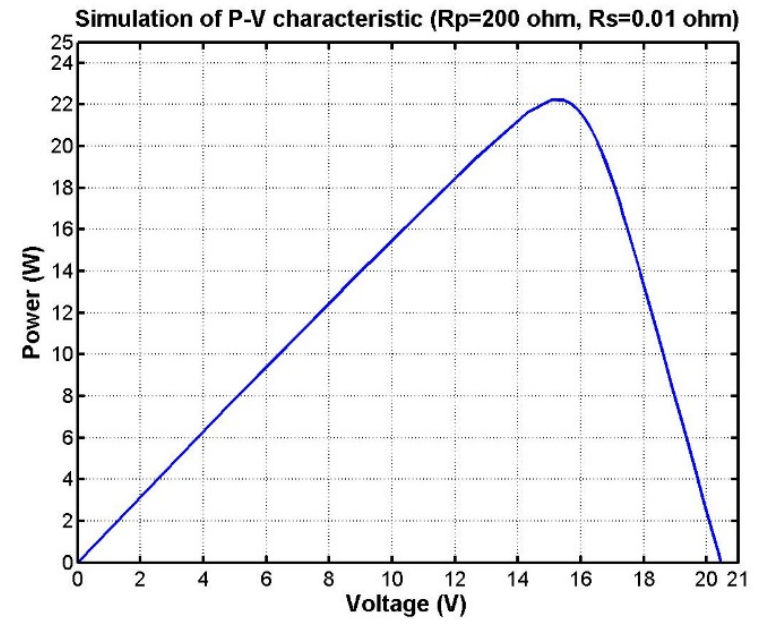

(a)

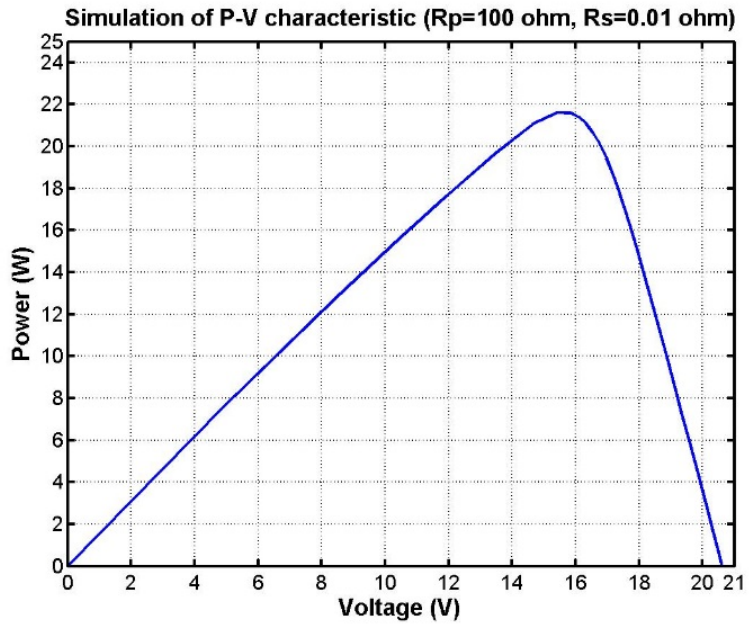

(b)

FIGURE 4. Simulation of $\mathrm{P}-\mathrm{V}$ characteristic with $\mathrm{Rp}=200 \Omega$ (a). Simulation with parallel resistance twice as low (b).

\section{Comparisons with experimental I-V characteristic}

For a scan duration equal to 0.1 second corresponding to the courant drop, data files shows a weak number of calculated points, equal to 34 for simulation with $\mathrm{Rp}=200 \Omega$ and 35 with $\mathrm{Rp}=100 \Omega$. It is to notice that to lengthen slope duration (1 or even 10 seconds) does not increase number of points.

Parameters set in each simulation like electrical variables deduced from data file are displayed in Table 2.

TABLE 2. Parameters and main electrical variables for first model simulations

\begin{tabular}{ccc}
\hline Parameters. Electrical variables & Simulation (a) & Simulation (b) \\
\hline Duration $(\mathrm{s})$ & 0.1 & 0.1 \\
$\mathrm{R}_{1}(\Omega)$ & $10.85 \mathrm{k}$ & $11.2 \mathrm{k}$ \\
$\mathrm{R}_{2}(\Omega)$ & $1 \mathrm{k}$ & $1 \mathrm{k}$ \\
$\mathrm{Rp}(\Omega)$ & 200 & 100 \\
$\mathrm{Rs}(\Omega)$ & 0.01 & 0.01 \\
$\mathrm{P}_{\text {peak }}(\mathrm{W})$ & 22.24 & 21.63 \\
$\mathrm{U}_{\text {mpp }}(\mathrm{V})$ & 15.06 & 15.42 \\
$\mathrm{I}_{\text {mpp }}(\mathrm{A})$ & 1.48 & 1.40 \\
$\mathrm{U}_{\text {oc }}(\mathrm{V})$ & 20.47 & 20.63 \\
\hline
\end{tabular}

When analyzing these results we must keep in mind that the decrease of Rp implies a drop of the open circuit voltage $\left(U_{o c}\right)$ if $R_{1}$ is not adjusted. In simulation (b) value of $R_{1}$ has risen but is clearly excessive because second $U_{o c}$ is a little higher.

To evaluate the most faithful simulated I-V characteristics, we report in Table 3 the relative errors obtained considering the experimental I-V characteristics.

TABLE 3. Relative variation for main electrical variables.

\begin{tabular}{ccc}
\hline Electrical variables & Simulation (a) & Simulation (b) \\
\hline$\Delta \mathrm{P}_{\text {peak }}(\%)$ & -10.0 & -12.9 \\
$\Delta \mathrm{U}_{\mathrm{mpp}}(\%)$ & -12.4 & -10.3 \\
$\Delta \mathrm{I}_{\mathrm{mpp}}(\%)$ & +2.7 & -2.8 \\
$\Delta \mathrm{U}_{\mathrm{oc}}(\%)$ & +0.3 & +1.1 \\
\hline
\end{tabular}

The two scenarios undervalue the power at MPP. For current, simulation (a) is a little optimistic, unlike simulation (b), and this last seems better thanks to a greater current slope between MPP and short circuit. With a Rp resistance between 100 and $200 \Omega$, for example $150 \Omega$, MPP current would certainly reach experimental value but it 
seems to be impossible to improve voltage at MPP, too low in all simulation cases. In addition, serial resistance Rs is very near from zero: greater values would reduce the MPP voltage. Besides, tests done with other short circuit currents $\left(\mathrm{I}_{\mathrm{sc}}\right.$ ), not shown in this paper, highlight an important and not rectifiable decrease of MPP voltage when $\mathrm{I}_{\mathrm{sc}}$ increases (Rs constant).

The main advantage of this model based on two Darlington circuit-mounted transistors is the realistic behavior around MPP. Nevertheless, as seen above, its main disadvantages are the impossibility to increase the current slope on the right side of the MPP for a fixed $\mathrm{I}_{\mathrm{sc}}$, a wrong voltage and a too low power at MPP with significant degradation when Isc rises. So this model, which possesses the advantage of simplicity, does not bring the expected robustness for a reliable simulation process of PV module.

\section{MODEL BASED ON A POWER TRANSISTOR CONTROLLED BY A ZENER DIODE}

\section{Electrical Diagram}

\section{Description}

As seen above, it is not possible with the model based on two Darlington circuit-mounted transistors to entirely adjust the current slope on the right side of the MPP. To improve the previous model, a solution consists to add a regulation of the voltage in front of the serial resistor Rs. In this case, when load dissipates the current, a voltage, easy to adjust with a resistance, will appear. A greater resistance increases the voltage drop, so the current slope can be less and less vertical on the right side of the MPP. At the opposite, reducing this resistance allows to increase the current slope. By this way the MPP voltage can be changed up to be closer to a realistic value. Such properties can be realized by a programmable Zener diode that sets a reference voltage on pin 1, Fig. 5 so that the current and the resultant voltage reach constant value in the voltage divider bridge $\left(\mathrm{R}_{1}-\mathrm{R}_{2}\right)$.

In open circuit, when there is no load current, the photo-generator current ( $\mathrm{I}_{\mathrm{CC}}$ in Fig.5) is bypassed with relatively low values by the divider bridge $R_{1}-R_{2}$, the parallel resistor $R p$, and the branch composed by the resistor $\mathrm{R}_{4}$ and the programmable Zener diode. The most important part is naturally bypassed by the PNP transistor. It is to note that a generic transistor has been chosen by convenience. $\mathrm{R}_{4}$ value is applied to limit the current in the diode at $100 \mathrm{~mA}$ maximum (for TL431) when transistor is ON.

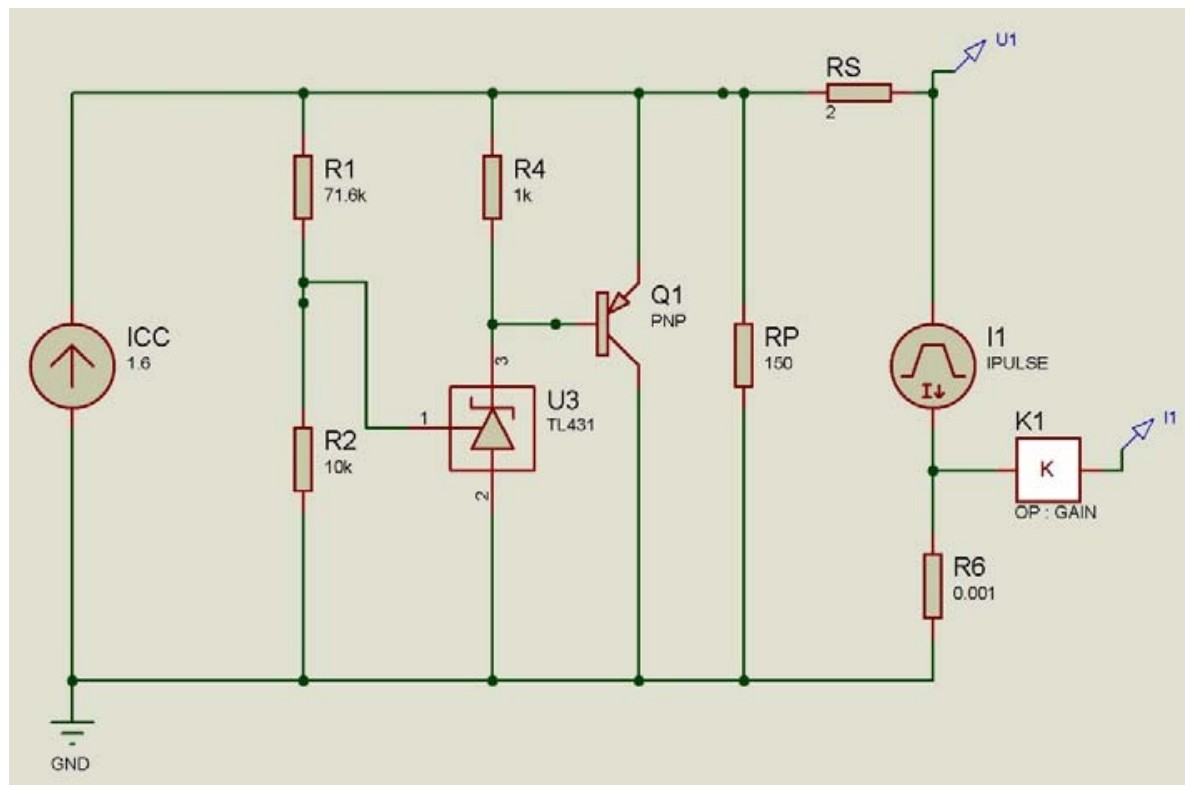

FIGURE 5. Model based on programmable Zener diode and power transistor 


\section{Open circuit voltage calculation}

The calculation of the open circuit voltage $\mathrm{U}_{\mathrm{co}}$ is much simpler than in the first model and it has the advantage not to depend on Rp. It is expressed by:

$$
U o c=\left(1+\frac{R 1}{R 2}\right) V r e f
$$

where $\mathrm{V}_{\text {ref }}$ is given by the Zener diode datasheet $(2.5 \mathrm{~V})$.

\section{Model behavior out of open circuit}

At the right of the MPP, the current passing through the transistor decreases linearly when the load current rises, knowing that current through $\mathrm{Rp}$ is constant thanks to voltage regulation. There is no effect of this resistance and the slope of PV module current is given by Rs. When the MPP is reached, the transistor becomes OFF, like the Zener diode connected to its base (pin 3, Fig. 5). Without regulation, the voltage is given by the current in $\mathrm{Rp}$ which decreases linearly implying a voltage drop down to zero in front of Rs. But, as the PV module voltage is measured behind this resistor, the model generates negative values at the end of simulation, due to voltage drop of Rs. The slope of the current at the left of the MPP depends on Rp. If Rp is high, the low initial current allows only a weak increase of the module current between the MPP and the short-circuit point.

\section{Simulations with Different Parameters}

\section{Setting of $R p$ and Rs resistances}

The two simulated I-V characteristics are reported with empirical values for serial and parallel resistances. Rp is equal to $300 \Omega$ in Fig. 6.a and equal to $150 \Omega$ in Fig. 6.b. It is to notice that compared to the $0.01 \Omega$ found with the first model, in this model, Rs becomes significant with a value of $2 \Omega$.

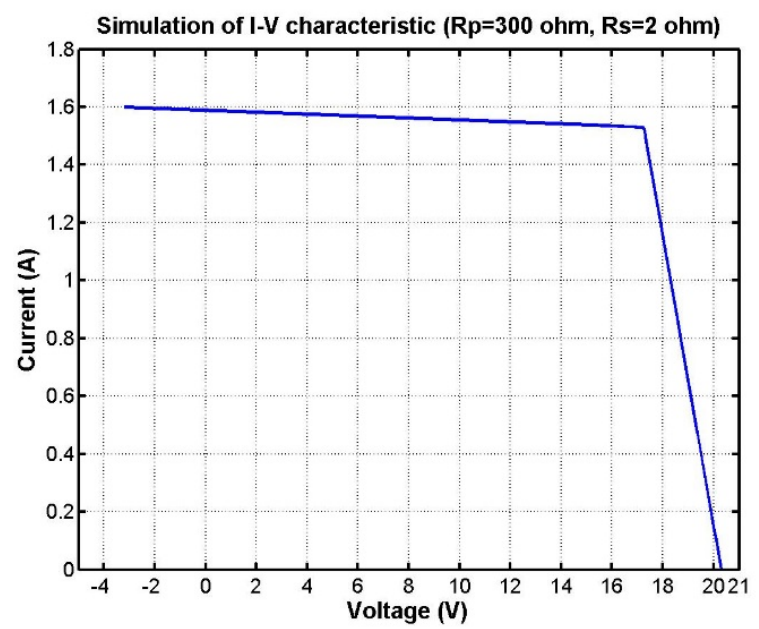

(a)

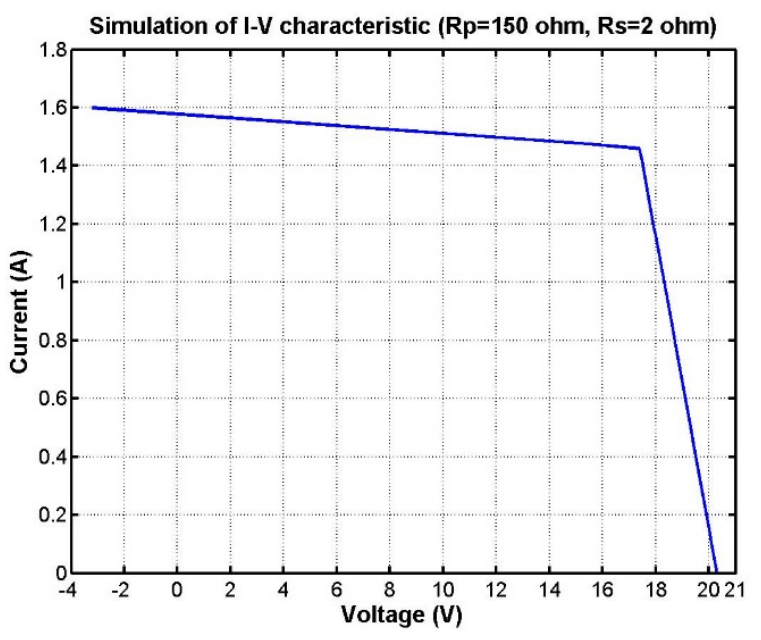

(b)

FIGURE 6. Simulation with parallel resistance equal to $300 \Omega$ (a). Simulation with parallel resistance twice as low (b). 


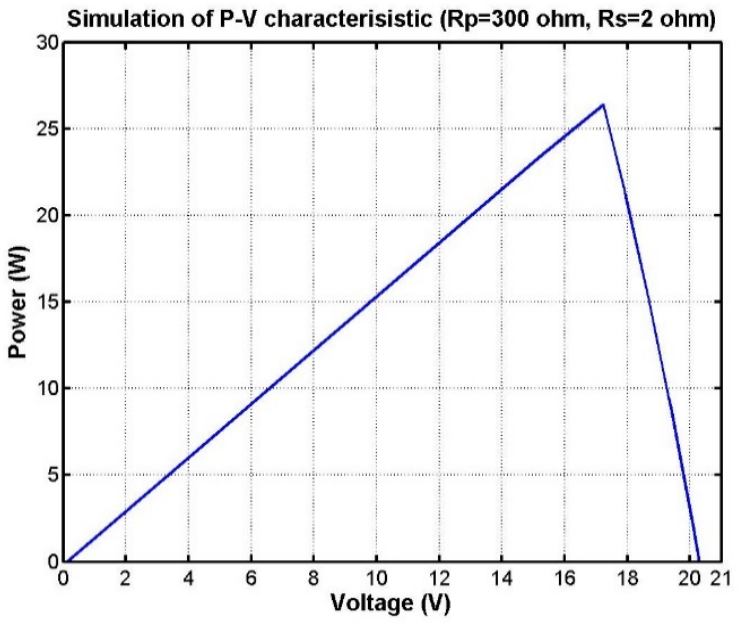

(a)

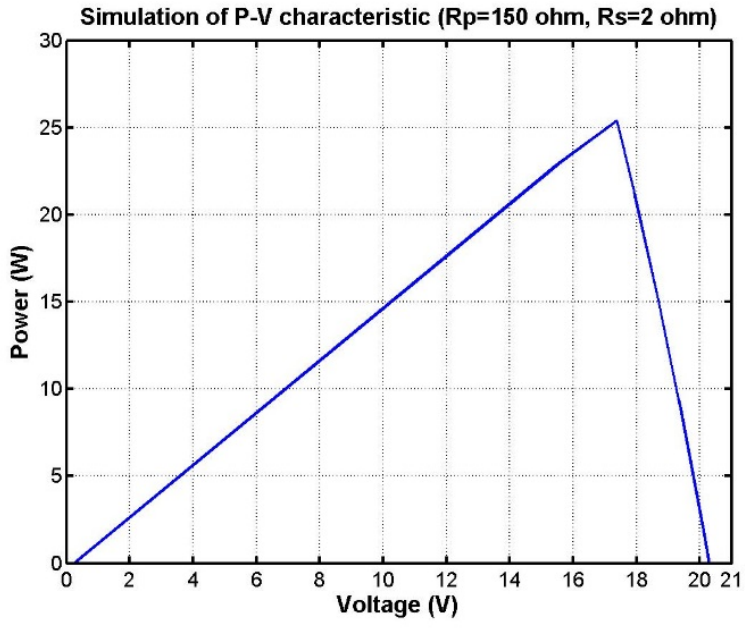

(b)

FIGURE 7. Simulation of $\mathrm{P}-\mathrm{V}$ characteristic with $\mathrm{Rp}=300 \Omega$ (a). Simulation with parallel resistance twice as low (b).

\section{Comparisons with experimental I-V characteristic}

In comparison with the model based on two Darlington circuit-mounted transistors, a quick scanning of data files shows a weaker number of calculated points, equal to 15 for simulation with $\mathrm{Rp}=300 \Omega$ with a duration of current slope equal to 0.1 second and equal to 13 with $\mathrm{Rp}=150 \Omega$ with a duration of current slope equal to 1 second. We can also notice that to lengthen or shorten slope durations $(0.1,1$ or even 10 seconds) do not modify the total number of points.

Parameters set in both simulations and like electrical variables deduced from data file are displayed in Table 4.

TABLE 4. Parameters and main electrical variables for second model simulations

\begin{tabular}{ccc}
\hline Parameters. Electrical variables & Simulation (a) & Simulation (b) \\
\hline Duration $(\mathrm{s})$ & 0.1 & 1.0 \\
$\mathrm{R} 1(\Omega)$ & $71.6 \mathrm{k}$ & $71.6 \mathrm{k}$ \\
$\mathrm{R} 2(\Omega)$ & $10 \mathrm{k}$ & $10 \mathrm{k}$ \\
$\operatorname{Rp}(\Omega)$ & 300 & 150 \\
$\operatorname{Rs}(\Omega)$ & 2.0 & 2.0 \\
$\mathrm{P}_{\text {peak }}(\mathrm{W})$ & 26.4 & 25.4 \\
$\mathrm{U}_{\text {mpp }}(\mathrm{V})$ & 17.24 & 17.38 \\
$\mathrm{I}_{\text {mpp }}(\mathrm{A})$ & 1.53 & 1.46 \\
$\mathrm{U}_{\mathrm{oc}}(\mathrm{V})$ & 20.31 & 20.31 \\
\hline
\end{tabular}

To calculate $\mathrm{R}_{1}$, formula (2) is considered with $\mathrm{R}_{2}$ equal to $10 \mathrm{k} \Omega$. For open circuit voltage, the value of $20.4 \mathrm{~V}$ found experimentally is applied.

In the last line of Table 4 it is to be noticed that simulated open circuit voltage is not the calculated value: it is a little lower than the experimental. Even if difference can be cancelled by $\mathrm{R}_{1}$ adjustment it is decided to keep theoretical value of $\mathrm{R}_{1}$.

To evaluate which is the most faithful setup within this model, relative errors are displayed in Table 5.

TABLE 5. Relative variation for main electrical variables.

\begin{tabular}{ccc}
\hline Electrical variables & Simulation (a) & Simulation (b) \\
\hline$\Delta \mathrm{P}_{\text {peak }}(\%)$ & +6.3 & +2.3 \\
$\Delta \mathrm{U}_{\mathrm{mpp}}(\%)$ & +0.2 & +1.0 \\
$\Delta \mathrm{I}_{\mathrm{mpp}}(\%)$ & +6.25 & +1.4 \\
$\Delta \mathrm{U}_{\mathrm{oc}}(\%)$ & -0.4 & $-0,4$ \\
\hline
\end{tabular}


Unlike the model based on two Darlington circuit-mounted transistors, this second model overvalues power at MPP but the relative difference is five times lower. Considering the current at the MPP, simulation (a) seems to be worse than the two simulations of the first model, but when considering the voltage at the same point, results are quite different. With the second model, this voltage is very close to the one measured experimentally. When integrating all calculations and comparisons, simulation (b) of the second model seems to be the best compromise for PV module simulation. Besides, perfect linear behavior of current on the both sides of the MPP allows a very easy calculation of parallel and serial resistances.

\section{Calculation of $R p$ and Rs resistances}

Simple application of Ohm's law leads to the two following formula:

$$
\begin{aligned}
& R_{s}=\frac{U o c-U M P P}{I M P P} \\
& R p=\frac{U M P P}{I s c-I M P P}
\end{aligned}
$$

Thanks to the experimental data, calculation gives respectively around $2.2 \Omega$ and $107.5 \Omega$ for serial and parallel resistances. With new parameters more realistic PV module characteristics can be simulated Fig.8, with truncating in second curve to avoid negative powers.

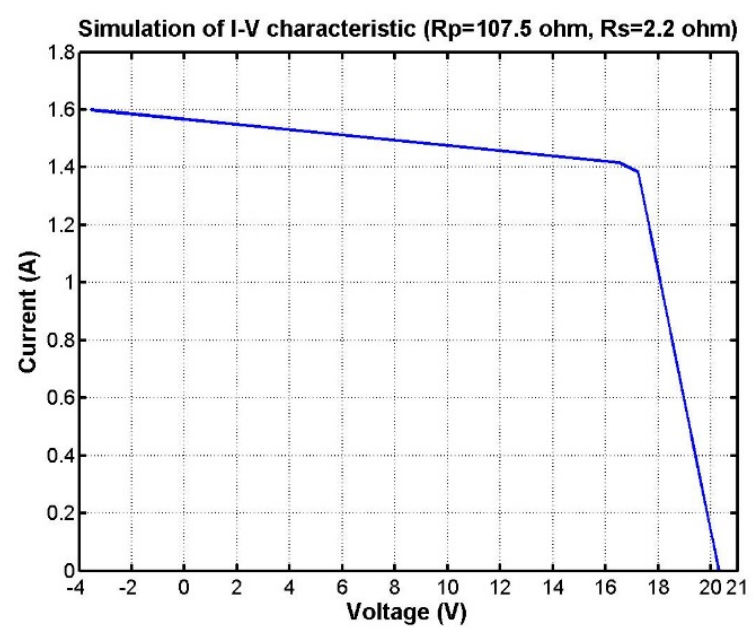

(a)

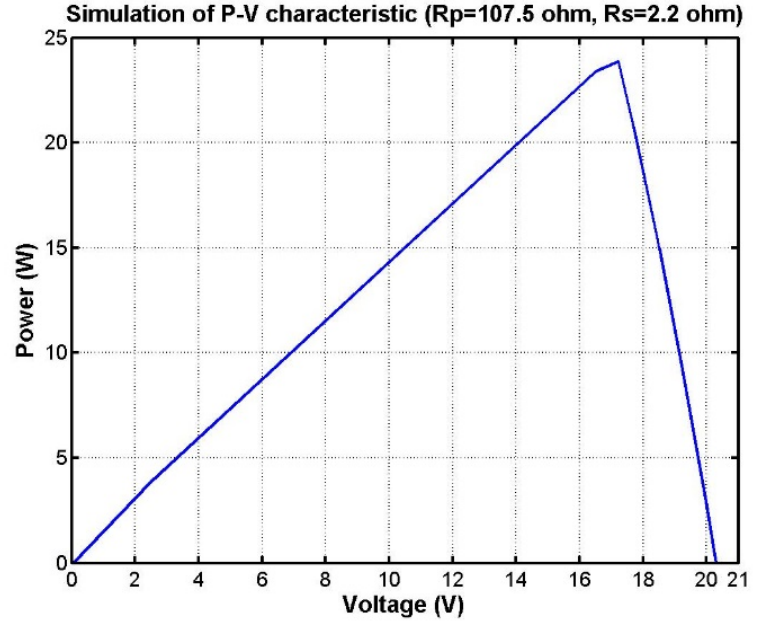

(b)

FIGURE 8. I-V characteristic with the calculated parameters (a). P-V characteristic with the calculated parameters (b).

The extraction of main electrical variables gives $\mathrm{P}_{\mathrm{MPP}}=23.87 \mathrm{~W}, \mathrm{U}_{\mathrm{MPP}}=17.23 \mathrm{~V}, \mathrm{I}_{\mathrm{MPP}}=1.385 \mathrm{~A}$ and $\mathrm{Uoc}=20.31 \mathrm{~V}$.

Considering the current for $0 \mathrm{~V}$ equal to $1.57 \mathrm{~A}$, as shown in Fig. 8.a, we remark there is only a little difference in comparison with the experimental short circuit value, which is equal to $1.6 \mathrm{~A}$. The presence of a negative voltage proves that the source current (Icc) in diagram Fig.5 is not sufficient. Thus, another simulation, shown in Fig.9, is tested with a new source current Icc equal to 1.63 A to compensate the Rs voltage drop inducing these negative voltages. 


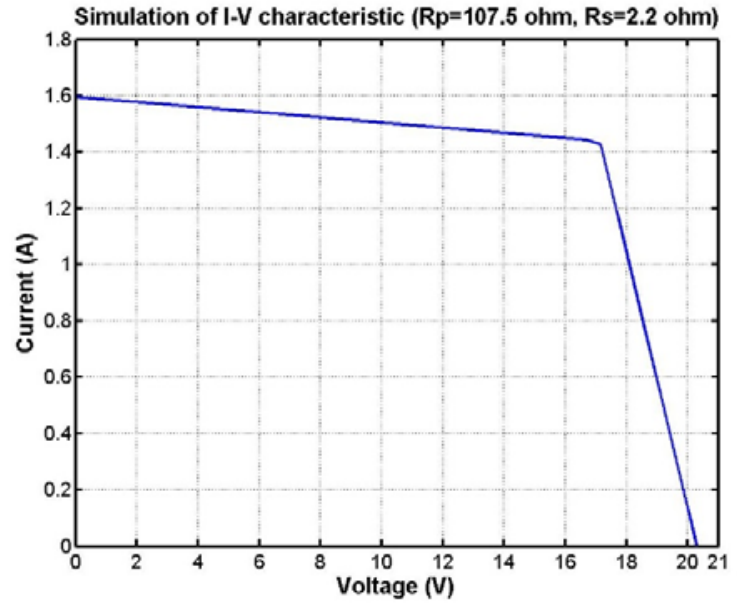

(a)

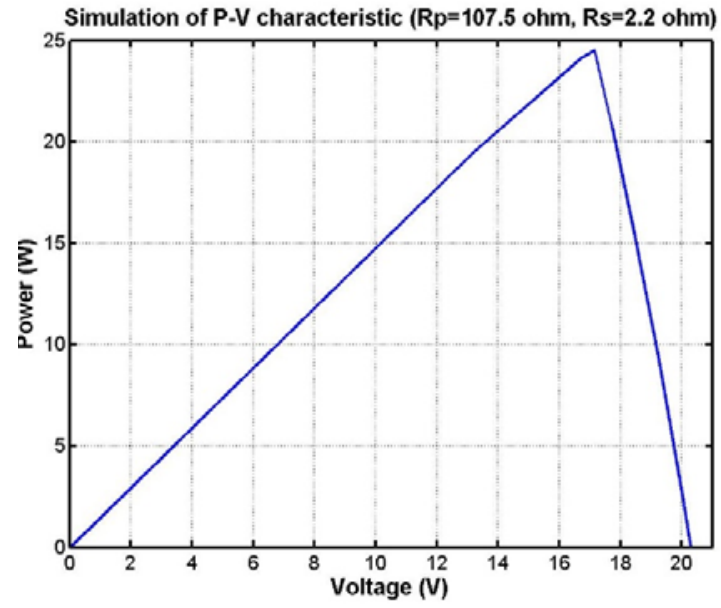

(b)

FIGURE 9. I-V characteristic with increased source current (a). P-V characteristic with increased source current (b).

The principal electrical characteristics are set in Table 6 and compared with experimental data. These results show that the improved model leads to results in accordance with experiment. Indeed, almost all the errors are under 1\%, which corresponds to very suitable results as it is less than the measurement errors done experimentally.

TABLE 6. Comparison between experiment and improved model.

\begin{tabular}{cccc}
\hline Electrical variables & Experiment & Simulation & Relative gap \\
\hline $\mathrm{P}_{\text {peak }}(\mathrm{W})$ & 24.83 & 24.525 & $-1.2 \%$ \\
$\mathrm{U}_{\text {mpp }}(\mathrm{V})$ & 17.20 & 17.16 & $-0.2 \%$ \\
$\mathrm{I}_{\mathrm{mpp}}(\mathrm{A})$ & 1.44 & 1.43 & $-0.7 \%$ \\
$\mathrm{I}_{\mathrm{sc}}(\mathrm{A})$ & 1.60 & 1.60 & $0 \%$ \\
$\mathrm{U}_{\mathrm{oc}}(\mathrm{V})$ & 20.40 & 20.31 & $-0.4 \%$ \\
\hline
\end{tabular}

\section{Modeling of PV module under Standard Test Conditions}

To test the reliability of this model based on a power transistor controlled by a Zener diode, we applied it for various irradiance levels and determined the simulated electrical variables when the PV module is illuminated under $1000 \mathrm{~W} / \mathrm{m}^{2}$. From the specifications of the PV module (see Table 1), the values of $\mathrm{R}_{1}, \mathrm{Rp}$ and Rs are calculated and equal to $77.2 \mathrm{k} \Omega, 58 \Omega$ and $1.48 \Omega$. After a preliminary simulation (not shown in this paper) to evaluate the correction to apply to the source current of the model, the characteristics I-V and P-V can be obtained. Results are reported in Fig.10 and principal electrical characteristics obtained by simulation are set in Table 7 and compared with PV module specifications (STC).

TABLE 7. Comparison between PV module specifications (STC) and improved model.

\begin{tabular}{cccc}
\hline Electrical variables & STC data & Simulation & Relative gap \\
\hline $\mathrm{P}_{\text {peak }}(\mathrm{W})$ & $50(50.75)$ & 50.40 & $+0.8 \%(-0.7 \%)$ \\
$\mathrm{U}_{\text {mpp }}(\mathrm{V})$ & 17.5 & 17.395 & $-0.6 \%$ \\
$\mathrm{I}_{\mathrm{mpp}}(\mathrm{A})$ & 2.9 & 2.898 & $-0.07 \%$ \\
$\mathrm{I}_{\mathrm{sc}}(\mathrm{A})$ & 3.2 & 3.2 & $0 \%$ \\
$\mathrm{U}_{\mathrm{oc}}(\mathrm{V})$ & 21.8 & 21.687 & $-0.5 \%$ \\
\hline
\end{tabular}




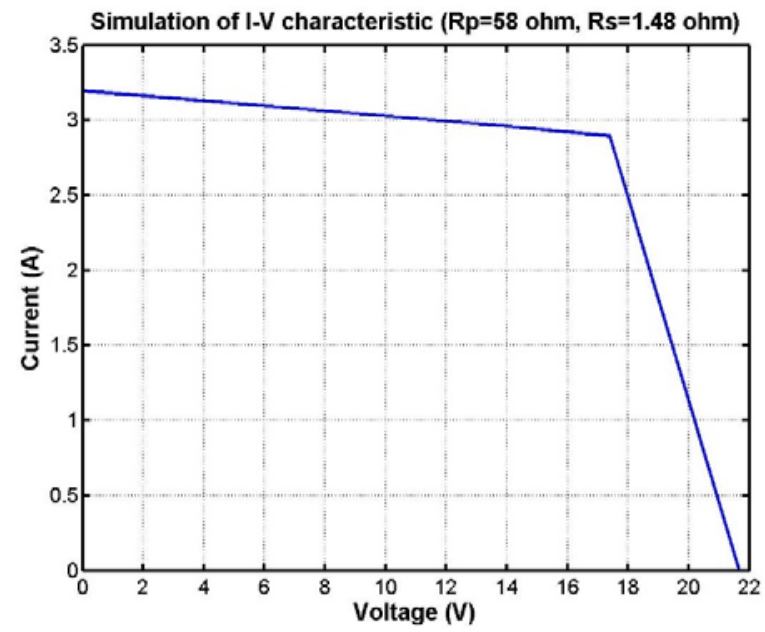

(a)

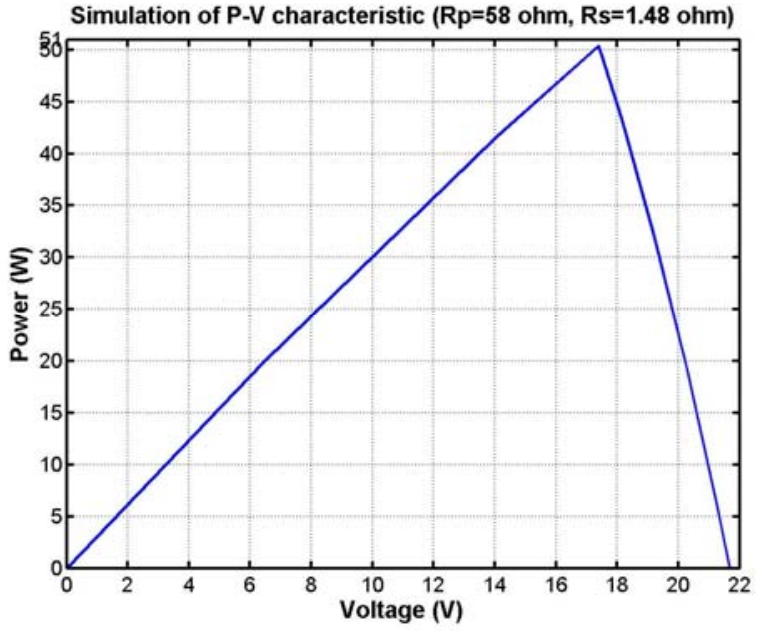

(b)

FIGURE 10. STC I-V characteristic with increased source current (a). STC P-V characteristic with increased source current (b).

We note that the maximum STC power is equal to $50 \mathrm{~W}$, but a simple calculation with current and voltage on this point give $50.75 \mathrm{~W}$ leading to an approximation in calculation. Finally, when this model is applied when the PV module is illuminated under $1000 \mathrm{~W} / \mathrm{m}^{2}$, (results reported in Table 7), we can formulate the same conclusions to those previously obtained (results reported in Table 6), i.e. a high reliability of the model which induces errors under $1 \%$, compared to the experimental validation.

In summary, the main advantages of the model based on a power transistor controlled by a Zener diode are an easy calculation of open circuit voltage, requiring only a little adjustment of the voltage divider bridge and realistic slopes of current on the both sides of the maximum power point. Nevertheless, it is to be noted that, when the voltage regulation stops, this model presents a rough behavior around the MPP.

The model needs calculation of three resistances $\left(R_{1}\right.$, Rs and $\left.R p\right)$ and two successive simulations to adjust source current in the electrical diagram.

Besides, if we consider in first approximation that open circuit and MPP voltages are quasi constant, in spite of irradiance variations, it would be interesting to verify if no excessive errors would be induced only modifying Rs, keeping Rp constant. So, this model could present a relative robustness to irradiance variations, with only two parameters to modify (Icc and Rs). Later simulations are planned to be presented in another paper to confirm or not this hypothesis.

It is planned to test the second model with other loads than current pulse. Preliminary simulations with magnetically coupled boost (MCB) converters have shown a good behavior of this structure, particularly around short circuit where there is no negative voltage any more. All detailed results will be published in a future contribution.

\section{CONCLUSION}

With the objectives to allow software simulation of complex photovoltaic architectures and to reduce the timeconsuming by calculations, we have presented two models of PV modules based on a minimal number of electronic components. The first model is based on two Darlington circuit-mounted transistors and the second one is based on a power transistor controlled by a Zener diode. Within the two models, the I-V characteristics were generated in very short time and with only few points pointing a good efficiency of both models. Nevertheless these two models give some discrepancy compared to experimental results. Indeed, in spite of a realistic behavior around the maximum power point, it is not possible, with first model, to obtain at this point the true voltage, implying a current slope at the right of this point too smooth to be realistic. Only current slope at the left of the MPP can be adjusted, choosing the good value of the parallel resistance. In this first model, the theoretical calculation of the open circuit voltage is done by keeping "standard" current gains of datasheets and by neglecting parallel resistance, which induces 
significant difference when testing. By else, in this first model, the dependence between the short circuit current used as source and resultant MPP is not adjustable. As a direct consequence, constituting a major drawback of this model, the MPP voltage drop is excessive when short circuit current rises, whereas, in the case of real PV module, the MPP voltage is found quasi constant in spite of variable irradiance.

At the opposite, the second model offers easy calculation of the open circuit voltage, and both current slopes on each side of the maximum power point are close to reality, near to the behavior observed experimentally. By else, this model is expected to show an essential quality to be proved in further studies, being certain robustness with irradiance changes, obtained with a single parameter, i.e. the serial resistance, needed to be adjusted.

\section{ACKNOWLEDGMENTS}

The authors gratefully acknowledge the Industrial Engineering and Maintenance (GIM) Department of the University Institute of Technology (IUT) of Thionville-Yutz, University of Lorraine (France), and particularly the Director, Dr. Patrick Klein for the financially support and for the facilities offered during these researches.

\section{REFERENCES}

1. T.T. Yetayew, T.R. Jyothsna, "Improved single-diode modeling approach for photovoltaic modules using data sheet”, IEE India Conference - INDICON (2013).

2. E. Gadjeva, M. Hristov, "Computer-aided model parameter extraction of photovoltaic modules using SPICE", in International Symposium Power Electronics Ee 2017, Serbia (2017).

3. S. Hosseini, S. Taheri, M. Farzaneh, H. Taheri, "An approach to precise modeling of photovoltaic modules under changing environmental conditions", IEEE Electrical Power and Energy Conference-EPEC, (2016).

4. M.H. El-Ahmar, A-H. El-Sayed, A.M. Hemeida, "Mathematical modeling of photovoltaic module and evaluation the effect of various parameters on its performance", in Power Systems Conference-MEPCON, IEEE (2016).

5. R. Mahto, P. Zarkesh-Ha, O. Lavrova, "MOSFET-Based modeling and simulation of Photovoltaics Module", in Photovoltaic Specialists Conference (PVSC), IEEE (2016).

6. P. Petit, A. Zegaoui, J.P. Sawicki, M. Aillerie, J.P. Charles, "New architecture for high efficiency DC-DC converter dedicated to photovoltaic conversion". Energy Procedia, 6, 688-694 (2011).

7. J.P. Sawicki, F. Saint-Eve, P. Petit, F. Mauffay, M. Aillerie, "PV Voltage Control in Spite of Disturbances on MCB Boost Output Voltage in Parallel Association", Energy Procedia, 119, 916-929 (2017).

8. J.P. Sawicki, P. Petit, A. Zegaoui, M. Aillerie, J.P. Charles, "High Efficiency Step-Up HVDC Converter for Photovoltaic Generator". Energy Procedia, 18, 1593-1600 (2012).

9. P. Petit, A. Zegaoui, J.P. Sawicki, M. Aillerie, J.P. Charles. "Rdson behavior in various MOSFET families", IEEE Industrial Electronics ISIE, 353-357 (2011).

10. A. Zegaoui, M. Aillerie, P. Petit, J.P. Charles, "Universal Transistor-based hardware SIMulator for real time simulation of photovoltaic generators". Solar Energy, 134, 193-201 (2016).

11. P. Petit, J.P. Sawicki, F. Saint-Eve, F. Maufay, M. Aillerie, "Optimized MPPT Algorithm for Boost Converters Taking Into Account the Environmental Variables". AIP Conference Proceedings 1758, 020015, 18 (2016). 